\title{
Robust far-field imaging by spatial coherence engineering
}

\author{
Yonglei $\mathrm{Liu}^{1,2}$, Yahong Chen ${ }^{3}$, Fei Wang ${ }^{3 *}$, Yangjian Cai1 ${ }^{1,2,3 *}$, \\ Chunhao Liang ${ }^{1,2 *}$ and Olga Korotkova ${ }^{4}$
}

The degree of coherence (DOC) function that characterizes the second-order correlations at any two points in a light field is shown to provide a new degree of freedom for carrying information. As a rule, the DOC varies along the beam propagation path, preventing from the efficient information recovery. In this paper, we report that when a partially coherent beam carrying a cross phase propagates in free space, in a paraxial optical system or in a turbulent medium, the modulus of the far-field (focal plane) DOC acquires the same value as it has in the source plane. This unique propagation feature is employed in a novel protocol for far-field imaging via the DOC, applicable to transmission in both freespace and turbulence. The advantages of the proposed approach are the confidentiality and resistance to turbulence, as well as the weaker requirement for the beam alignment accuracy. We demonstrate the feasibility and the robustness of the far-field imaging via the DOC in the turbulent media through both the experiment and the numerical simulations. Our findings have potential applications in optical imaging and remote sensing in natural environments, in the presence of optical turbulence.

Keywords: degree of coherence; cross phase; optical imaging

Liu YL, Chen YH, Wang F, Cai YJ, Liang CH et al. Robust far-field imaging by spatial coherence engineering. Opto-Electron Adv 4, 210027 (2021).

\section{Introduction}

Optical coherence state is one of the intrinsic characteristics of a partially coherent beam (PCB), describing the electric field correlation at pairs of spatial locations and times $^{1}$. In the space-frequency domain, spatial coherence properties are characterized by the two-point spectral degree of coherence (DOC) function, being a normalized version of the cross-spectral density (CSD) function. Over past several decades, the PCBs have attracted substantial academic attention owing to their capability to resist turbulence-induced negative effects and to reduce the speckle noise, and therefore, they have found important applications in free-space optical communications, laser materials processing, inertial confinement fusion, optical imaging, etc. ${ }^{1-6}$. The Schell-model beams whose DOC is only a function of the separation of two spatial points are the most commonly encountered PCBs. They were extensively studied in the past few decades. However, these studies were mainly limited to Gaussian Schell-model beams, i.e., those with the DOC

\footnotetext{
${ }^{1}$ Shandong Provincial Engineering and Technical Center of Light Manipulations \& Shandong Provincial Key Laboratory of Optics and Photonic Device, School of Physics and Electronics, Shandong Normal University, Jinan 250014, China; ${ }^{2}$ Collaborative Innovation Center of Light Manipulations and Applications, Shandong Normal University, Jinan 250358, China; ${ }^{3}$ School of Physical Science and Technology, Soochow University, Suzhou 215006, China; ${ }^{4}$ Department of Physics, University of Miami, Coral Gables, Florida 33146, USA.

"Correspondence: F Wang, E-mail: fwang@suda.edu.cn; YJ Cai, E-mail: yangjiancai@suda.edu.cn; CH Liang, E-mail: Cliang@dal.ca

Received: 25 February 2021; Accepted: 6 June 2021; Published online: 24 December 2021
}

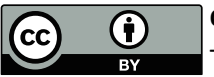

Open Access This article is licensed under a Creative Commons Attribution 4.0 International License

To view a copy of this license, visit http://creativecommons.org/licenses/by/4.0/.

(C) The Author(s) 2021. Published by Institute of Optics and Electronics, Chinese Academy of Sciences. 
having a Gaussian profile. One of the difficulties for constructing a physically reliable PCB with non-Gaussian, and more generally non-Schell-like correlations, is that the CSD function must satisfy some constraints such as Hermiticity and non-negative definiteness. In 2007, Gori and co-workers applied the Bochner theorem of functional analysis for suggesting a sufficient and necessary condition to devise genuine CSD functions ${ }^{7}$ (see ref. ${ }^{8}$ for its vectorial generalization). Since then, the extensive work has been devoted to devising PCBs with different kinds of DOCs ${ }^{9-11}$, propagation characteristics and interaction with matter ${ }^{12-20}$. In particular, it was established that the DOC can be used as a new degree of freedom to control the free-space beam evolution and its interactions with linear media to reduce the turbulence-induced effects, and so on. Consequently, the PCBs with the prescribed DOC have recently become promising candidates in diverse applications including self-reconstruction, super resolution imaging, diffractive imaging and optical encryption ${ }^{21-29}$. However, the DOC is not propagation-invariant which hinders its use in technologies requiring optical information transfer and recovery. The general problem of modeling of the PCBs whose DOC remains propagation invariant has not yet been tackled.

High-resolution image recovery is a complex problem that has been classically approached by means of the optical signal's average intensity manipulation $s^{30}$. In general, amplitude, phase, polarization, spectrum and other parameters of the wave-field are available for imaging or image acquisition ${ }^{25-34}$. Nevertheless, in some practical situations such as in the presence of turbulence or turbidity, the amplitude and phase are susceptible to the influence of turbulence ${ }^{35}$. The image information contained in the amplitude or in the phase becomes lost with propagation range. Hence, one has to explore other parameters for transmitting/retrieving image information. In fact, it was shown in recent studies ${ }^{36-38}$ that the modulus of the DOC of the field scattered from a turbid medium is an efficient parameter that can be used to retrieve the embedded object's information. However, since the DOC changes on propagation, it appears to be applicable only for short-distance image reconstruction.

In this article, we report that in situations when a cross phase $(\mathrm{CP})$ is imposed on a $\mathrm{PCB}$, the far-field modulus of its DOC propagating through free space, a paraxial optical system, and atmospheric turbulence returns to its value in the source plane. This finding is demonstrated through numerical examples and experiments. Further, based on the peculiar recovery feature of the DOC, an efficient approach is proposed for encoding the image information into the DOC profile and its transmission through both free space and atmospheric turbulence. The obtained image is, indeed, shown to be resistant to the negative effects induced by turbulence. This paper is organized as follows. First, the effect of the $\mathrm{CP}$ on the DOC of a PCB during propagation is studied theoretically. Next, the experiment is carried out to demonstrate the recovery of the far-field DOC in free space and in atmospheric turbulence. Then, a new scheme for the transmission of image via the DOC through the free space and turbulence is studied and the robustness of far-field imaging is demonstrated. Finally, the results are summarized.

\section{The effect of a Cross Phase on the DOC of partially coherent beams}

Suppose a scalar, quasi-monochromatic Schell-model beam carrying a $\mathrm{CP}$, propagates along the $z$-axis. In the source plane $(z=0)$, the CSD function of such a beam has the following form ${ }^{12}$

$$
W_{0}\left(\boldsymbol{r}_{1}, \boldsymbol{r}_{2}\right)=\tau\left(\boldsymbol{r}_{1}\right) \tau^{*}\left(\boldsymbol{r}_{2}\right) \mu_{0}(\Delta \boldsymbol{r}) \exp \left[\mathrm{i} u\left(x_{1} y_{1}-x_{2} y_{2}\right)\right],
$$

where $\boldsymbol{r}_{i}=\left(x_{i}, y_{i}\right), i=1,2$ denote two arbitrary transverse position vectors, perpendicular to the $z$-axis; $\Delta r=r_{1}-r_{2}=$ $(\Delta x, \Delta y)$ is the difference of two position vectors and $\tau(\boldsymbol{r})$ is a complex amplitude function. For simplicity, we assume that the amplitude function is Gaussian: $\tau(\boldsymbol{r})=\exp \left(-\boldsymbol{r}^{2} / 4 \omega_{0}^{2}\right)$ with $\omega_{0}$ being the beam width while $\mu_{0}(\Delta r)$ is the DOC, which only depends on the difference of $\boldsymbol{r}_{1}$ and $\boldsymbol{r}_{2}$. The last term $\exp \left[\mathrm{i} u\left(x_{1} y_{1}-x_{2} y_{2}\right)\right]$ is the $\mathrm{CP}$, where $u$ is a measure of the strength of the CP. The sign of the parameter $u$ only determines the rotation direction of the beam ${ }^{39}$. It can be regarded as the extension of astigmatic phase, and it is a separable function with respect to $\left(x_{1}, y_{1}\right)$ and $\left(x_{2}, y_{2}\right)$. Due to the coupling of the $x$ and $y$ in the CP, the beam will exhibit the particular distribution orbital angular momentum (OAM) flux density along the propagation direction, which is responsible for the rotation of beam spot during propagation. The CP has been extensively explored ${ }^{39-44}$. The studies reveal that such a phase structure can make the beam rotate ${ }^{39}$, realize coherent modes conversion ${ }^{41}$, flexibly focus the ring Pearcey beam ${ }^{42}$, as well as manipulate the high-order optical vortices ${ }^{43,44}$. 
In order to investigate the effect of the CP on the evolution of the DOC, we first set $\mu_{0}(\Delta r)$ as the cosine-Gaussian correlated Schell-model (CGCSM) source ${ }^{16}$

$$
\mu_{0}(\Delta \boldsymbol{r})=\cos \left(\frac{n \sqrt{2 \pi} \Delta x}{\delta_{0}}\right) \cos \left(\frac{n \sqrt{2 \pi} \Delta y}{\delta_{0}}\right) \exp \left(-\frac{\Delta \boldsymbol{r}^{2}}{2 \delta_{0}^{2}}\right),
$$

where $\delta_{0}$ denotes the source coherence width and $n$ is the beam order. Within the regime of paraxial approximation, the relationship between the CSD function in the source plane and the receiver plane containing a generic paraxial $\mathrm{ABCD}$ optical system is given by the Collins formula $^{14}$

$$
\begin{aligned}
& W\left(\boldsymbol{\rho}_{1}, \boldsymbol{\rho}_{2}\right)=\frac{k^{2}}{4 \pi^{2} \boldsymbol{B}^{2}} \exp \left[-\frac{\mathrm{i} k \boldsymbol{D}}{2 \boldsymbol{B}}\left(\boldsymbol{\rho}_{1}^{2}-\boldsymbol{\rho}_{2}^{2}\right)\right] \\
& \times \int_{-\infty}^{\infty} \int_{-\infty}^{\infty} W_{0}\left(\boldsymbol{r}_{1}, \boldsymbol{r}_{2}\right) \exp \left[-\frac{\mathrm{i} k \boldsymbol{A}}{2 \boldsymbol{B}}\left(\boldsymbol{r}_{1}^{2}-\boldsymbol{r}_{2}^{2}\right)\right] \\
& \times \exp \left[\frac{\mathrm{i} k}{\boldsymbol{B}}\left(\boldsymbol{r}_{1} \cdot \boldsymbol{\rho}_{1}-\boldsymbol{r}_{2} \cdot \boldsymbol{\rho}_{2}\right)\right] \mathrm{d}^{2} \boldsymbol{r}_{1} \mathrm{~d}^{2} \boldsymbol{r}_{2},
\end{aligned}
$$

where $k=2 \pi / \lambda$ stands for the wavenumber with wavelength $\lambda, \rho_{1}$ and $\rho_{2}$ denote two transverse position vectors in the receiver plane, and $\boldsymbol{A}, \boldsymbol{B}$ and $\boldsymbol{D}$ are the elements of the optical system's transfer matrix. Let us consider a single-lens focusing system: a thin lens with focal length $f$ is placed in the source plane followed by freespace path of distance $z$ after the lens. Under this circumstance, the elements of the transfer matrix are found from the $A B C D$ matrix multiplication as

$$
\left(\begin{array}{ll}
\boldsymbol{A} & \boldsymbol{B} \\
\boldsymbol{C} & \boldsymbol{D}
\end{array}\right)=\left(\begin{array}{ll}
1 & z \\
0 & 1
\end{array}\right)\left(\begin{array}{cc}
1 & 0 \\
-1 / f & 1
\end{array}\right)=\left(\begin{array}{cc}
1-z / f & z \\
-1 / f & 1
\end{array}\right) .
$$

On substituting Eqs. (1), (2) and (4) into Eq. (3) and after integration, we obtain the analytical expression for the CSD function in the receiver plane (see supplementary material). Interestingly, it is found that in the focal plane ( $z=f$, namely, in the far field), the modulus of the DOC reduces to the following form

$\left|\mu_{f}(\Delta \rho)\right|=\left|\cos \left(\frac{n \sqrt{2 \pi} \Delta \rho_{x}}{\delta_{f}}\right) \cos \left(\frac{n \sqrt{2 \pi} \Delta \rho_{y}}{\delta_{f}}\right) \exp \left(-\frac{\Delta \rho^{2}}{2 \delta_{f}^{2}}\right)\right|$,

where $\delta_{f}=(f u / k) \delta_{0}$ is the coherence width in the focal plane and $\Delta \boldsymbol{\rho}=\boldsymbol{\rho}_{1}-\boldsymbol{\rho}_{2}=\left(\Delta \rho_{x}, \Delta \rho_{y}\right)$, provided that factor $u$ satisfies condition

$$
u>\max \left\{\sqrt{400 \pi n^{2} / \delta_{0}^{2}-\Omega^{2}} / \omega_{0}, \sqrt{10} \Omega / \omega_{0}\right\},
$$

where $\Omega^{2}=1 / 4 \omega_{0}^{2}+1 / \delta_{0}^{2}$. On comparing Eq. (5) to Eq. (2), we establish that the modulus of the DOC in the focal plane has the same form as that in the source plane but a different value of the coherence width. This peculiar propagation feature is quite different from that of the CGCSM beam without the CP, whose DOC in the focal plane degenerates to other forms. In general, the DOC itself can be complex already at the source plane due to the effects of the initial CP, and/or may become even more complex during propagation. Nevertheless, the modulus of the DOC in the focal region returns to the same form as it is in the source plane. In Fig. 1 the evolution of $|\mu|$ with propagation distance $z$, for different strength factors is presented. The first row is set for comparison, for the case of trivial CP, i.e., $u=0$. In the absence of CP or when factor $u$ is small $\left(u=2 \mathrm{~mm}^{-2}\right)$, the modulus of the DOC gradually transforms to other shapes near the focal region [see first and second rows]. As the value of strength factor reaches $10 \mathrm{~mm}^{-2}$ or higher [while satisfying Eq. (6)], it can be seen that the modulus of the DOC near the focal plane indeed returns to that in the source plane. Note that the DOC is twisted and rotates during propagation because of the CP's presence [see Fig. 1(c2) and $1(\mathrm{~d} 2)$ ]. In fact, the DOC in the focal plane rotates $\pi / 2 \mathrm{rad}$, but its shape is unchanged. The rotation of the DOC attributes to the particular orbital angular momentum (OAM) flux density induced by the $\mathrm{CP}^{39}$.

Although in the preceding analysis, a specific example is used for $\mu_{0}(\Delta \boldsymbol{r})$, the discovered phenomenon of the recovery of the DOC's modulus in the focal plane (far field) can be generalized to any Schell-model beam carrying the $\mathrm{CP}$. To justify this claim, a rather general alternative form of the DOC representing the Fourier transform of letter " $\mathrm{S}$ " (known as the power spectral density) is chosen as the example, [see the top right corner of Fig. 2(a)]. In this case, the DOC is a complexvalued function not assuming any analytical expression. The numerical result for the modulus of the DOC in the source plane is shown in Fig. 2(a). When such a beam carrying a CP with strength factor $u=-60 \mathrm{~mm}^{-2}$ propagates through the focusing system, the modulus of the DOC distribution undergoes shaping and rotating [see in Fig. 2(b) and 2(c)]. It gradually turns back to the initial form, with the expected 90-degree pattern rotation [see in Fig. 2(d)] in the focal plane. The examples based on other Schell-model sources carrying the CP (not shown here) also confirm this far-field DOC's modulus recovery mechanism. In addition, the focusing lens does not need to be placed in the source plane of the beam. In fact, 

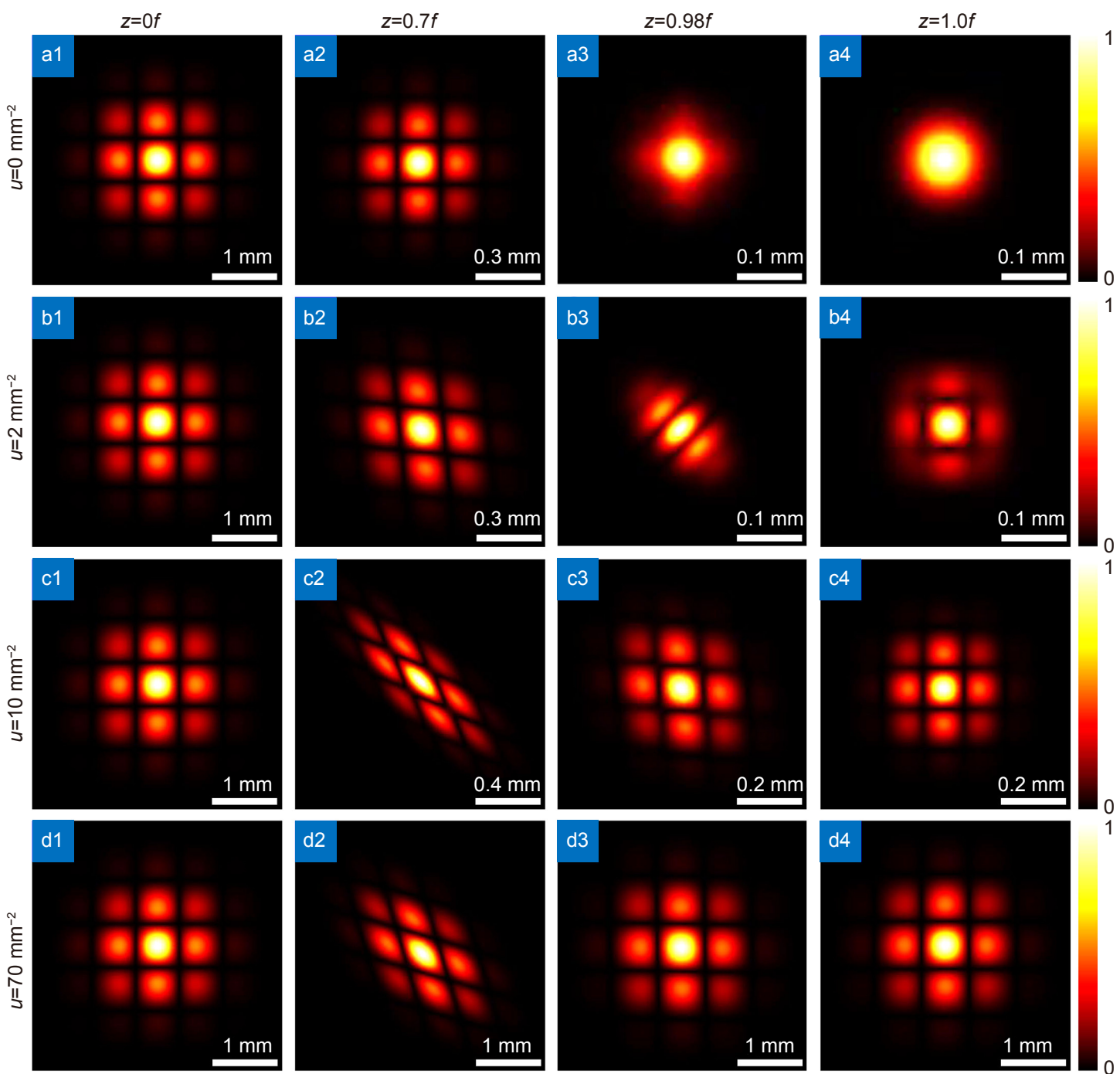

Fig. 1 | Variation of the modulus of DOC $|\mu|$ of a CGCSM beam with the propagation distance $z$ after the focusing lens, with the CP strength factor (a1-a4) $u=0 \mathrm{~mm}^{-2},(\mathbf{b} 1-\mathbf{b} 4) u=2 \mathrm{~mm}^{-2},(\mathbf{c} 1-\mathbf{c} 4) u=10 \mathrm{~mm}^{-2}$, and (d1-d4) $u=70 \mathrm{~mm}^{-2}$. The parameters are set as $\lambda=532$ $\mathrm{nm}, \delta_{0}=0.5 \mathrm{~mm}$ and $n=1$.
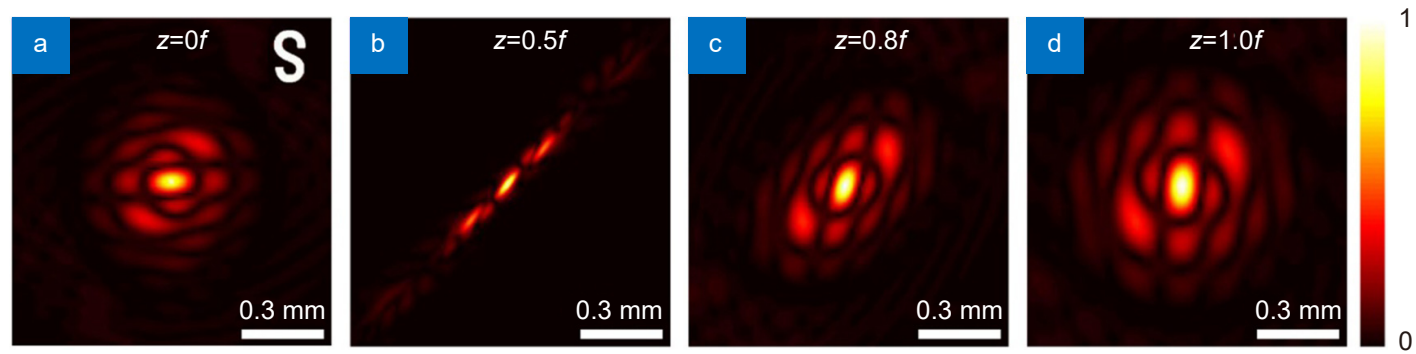

Fig. 2 | Theoretical results of the modulus of the DOC $|\mu|$ at different propagation distances $z$ after the lens with the CP strength factor $\boldsymbol{u}=-\mathbf{6 0} \mathrm{mm}^{-2}$. The inserted letter " $\mathrm{S}$ " in (a) is adopted as the power spectral density $P(\boldsymbol{v})$ function.

it can be placed in any transverse propagation plane $z=$ const. And in the focal plane of the lens, the modulus of the DOC is also the same as that in the source plane $(z=$ 0 ), irrespective of the DOC distribution at the plane where the focusing lens is placed.
Recovery of the modulus of the DOC of Schell-model beams with a CP during propagation: experimental verification

In this section, we carry out the experiment to demonstrate the results obtained in the previous section. Figure 3 


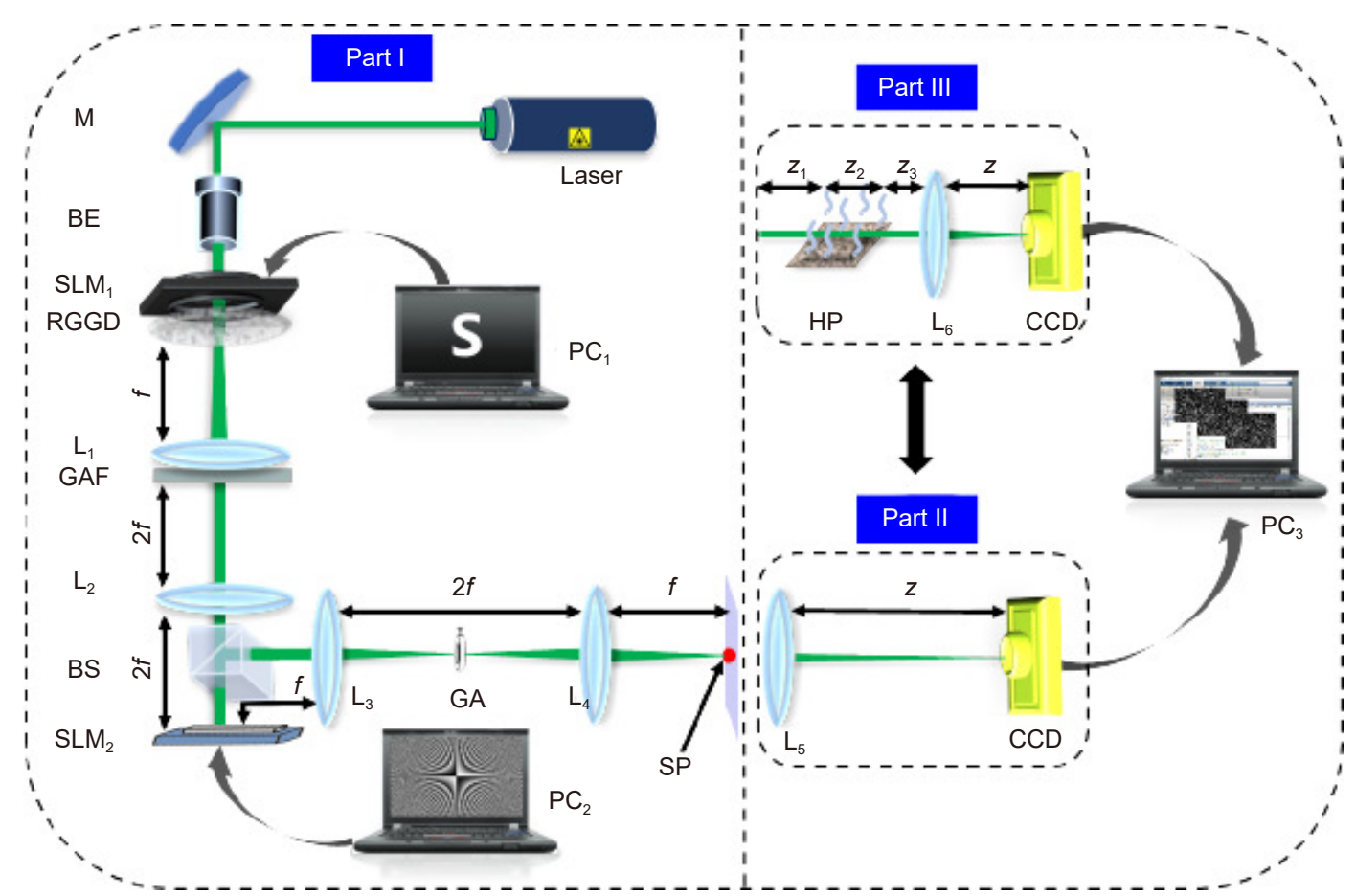

Fig. 3 | Schematic diagram of the experiment setup for generation of Schell-model beams with a controllable CP structure, measurement of the modulus of the DOC in the far field propagation in free space as well as in turbulent atmosphere. Laser, a Nd:YAG laser with wavelength 532nm; $M$, mirror; $B E$, beam expander; $S_{2} M_{1}, S L M_{2}$, spatial light modulator; $R G G D$, rotating ground glass disk; $L_{1}, L_{2}, L_{3}, L_{4}, L_{5}, L_{6}$, thin lenses with the identical focal length $f=250 \mathrm{~mm}$; GAF, Gaussian amplitude filter; BS, beam splitter; CA, circular aperture; SP, source plane; $\mathrm{CCD}$, charge-coupled device; $\mathrm{HP}$, hot plate; $\mathrm{PC}_{1}, \mathrm{PC}_{2}, \mathrm{PC}_{3}$, personal computers.

illustrates the schematic diagram for the experimental setup. It includes three parts. Part I is the optical system for the generation of a Schell-model beam with a CP. A laser beam emitted from a Nd:YAG laser $(\lambda=532 \mathrm{~nm})$ is reflected by a mirror and expanded by a beam expander $(\mathrm{BE})$, then arrives at a spatial light modulator $\left(\mathrm{SLM}_{1}\right)$. A computer-generated hologram is loaded on the $\mathrm{SLM}_{1}$ which is used to produce a prescribed intensity pattern on the front surface of a Rotating Ground Glass Disk (RGGD). The scattered light is then collimated by a Lens $\mathrm{L}_{1}$ and is transformed from the uniform light intensity to a Gaussian distribution by a Gaussian amplitude filter (GAF). If the size of the intensity pattern on the RGGD is much larger than the inhomogeneity scale of the RGGD, the DOC of the beam in the GAF plane can be described as the following integral according to the Van-Cittert Zernike theorem

$$
\mu(\Delta \boldsymbol{r}) \propto \int P(\boldsymbol{v}) \exp (-\mathrm{i} 2 \pi \boldsymbol{v} \cdot \Delta \boldsymbol{r} / \lambda f) \mathrm{d}^{2} \boldsymbol{v},
$$

where $P(v)$ is the intensity distribution in the RGGD plane, also known as the power spectral density in the literatures $^{15-17}$. The generated partially coherent light beam is then imaged onto a $\mathrm{SLM}_{2}$ screen with unit magnification through a Lens $\mathrm{L}_{2}$. The $\mathrm{CP}$ associated with a phase shift, i.e., $\varphi(x, y)=u x y+2 \pi f_{x} x$, where $f_{x}$ is the spatial fre- quency along $x$ direction, is computed as a blaze phase grating loaded on the $\mathrm{SLM}_{2}$. The reflected light from $\mathrm{SLM}_{2}$ passes through a $4 f$ optical imaging system consisting of Lens $\mathrm{L}_{3}$ and $\mathrm{L}_{4}$, where a circular aperture is inserted in its Fourier spectrum plane to filter out the first-order diffraction. In the output plane, the PCB with the CP is then synthesized.

The detection system is shown in the part II. A focusing lens $\mathrm{L}_{5}$ collects the incident light beam and the $\mathrm{CCD}$ is located in the focal plane to measure the modulus of the DOC. The measurement procedures are carried out as follows: the CCD first records a series of instantaneous light intensity images in the chronological order. Based on the Gaussian momentum theorem, the square of the modulus of the DOC is calculated using the following formula

$$
\left|\mu\left(x-x_{0}, y-y_{0}\right)\right|^{2}=\frac{\sum_{n=1}^{N} I\left(x_{0}, y_{0}, t_{n}\right) I\left(x, y, t_{n}\right)}{N \bar{I}\left(x_{0}, y_{0}\right) \bar{I}(x, y)}-1,
$$

where $N$ is the total number of frames captured by the CCD. $I\left(x, y, t_{n}\right)$ denotes the recorded instantaneous intensity at point $(x, y)$ and time $t_{n} . \bar{I}(x, y)=\sum_{n=1}^{N}$ $I\left(x, y, t_{n}\right) / N$ represent the time average intensity at point 
$(x, y) .\left(x_{0}, y_{0}\right)$ is the reference point. Owing to that the DOC only depends on the separation of two spatial points, the choice of the reference point can be arbitrary. In addition, if the coherence width $\delta_{0}$ is sufficiently small, the autocorrelation function of a single instantaneous intensity converges to the square of the modulus of the DOC, which substantially saves time to acquire the correlation function instead of time average.

In Fig. 4, the experimental results for $|\mu|$ in the focal plane with different strength factors at different propagation distances after Lens $L_{5}$ are presented. The power spectral density (intensity distribution in the RGGD plane) is taken as letter " $\mathrm{S}$ ", and $\mathrm{L}_{5}$ is located in the source plane. Without the CP, the DOC in the focal plane degenerates to a Gaussian-like spot [see Fig. 4(a)]. As the strength factor $|u|$ increases, the DOC becomes greatly affected by the CP [see Fig. 4(b) and 4(c)]. As expected, the DOC pattern gradually returns to that in the source plane [see in Fig. 2(d) for theoretical calculation and in Fig. 4(d) for experimental results], when the strength factor $u$ is $-40 \mathrm{~mm}^{-2}$ or $|u|$ is higher. During the focusing process, the DOC pattern distorts, rotates, and finally again becomes the same as in the source plane, which implies that the DOC recovers only near the focal plane (in the far field). The experimental results reasonably agree with the theoretical calculations shown in Fig. 2. However, the background noise level is relatively high in the experimental results. One possible reason for it is that the measured DOC is evaluated from the statistical properties of the instantaneous intensity, using only a finite number of samples ( $N=3000$ in the experiment). As a result, the DOC represents a slowly converging distribution; hence, the fluctuations occur in the data processing. Another reason is that as shown in Eq. (8), the measured quantity in the experiment is the square of the modulus of the DOC and not the modulus of the DOC itself. In the calculation of the square root, the background noise is greatly amplified, which may be one of the main reasons for the high background noise. Besides, other unexpected sorts of noise may be resulting from random processes relating to light modulation by the RGGD and the SLM.

As already mentioned above, one advantage of the PCBs used as the information carrier in the optical communication systems stems from the fact that they are less susceptible to the turbulence-induced negative effects such as beam wander, intensity scintillation and de-coherence, as compared to the coherent beams. To study the effects of turbulence on the DOC, the experimental setup shown in part III of Fig. 3 is established. The generated beam with the $\mathrm{CP}$ first propagates at range $z_{1}=$ $0.5 \mathrm{~m}$. Then, it travels just above a hot plate (HP) with side $z_{2}=0.3 \mathrm{~m}$ placed for mimicking the atmospheric turbulence through convection. Finally, it arrives at the detection plane after passing at distance $z_{3}=0.2 \mathrm{~m}$ beyond the HP. The HP controls the strength of turbulence by adjusting its temperature setting. The experimental results shown in Fig. 5 illustrate that the modulus of the DOC in the focal plane remains almost invariant for different strength settings of turbulence [see Fig. 5(b)-5(d)], implying that the DOC's modulus is little sensitive to the atmospheric turbulence.
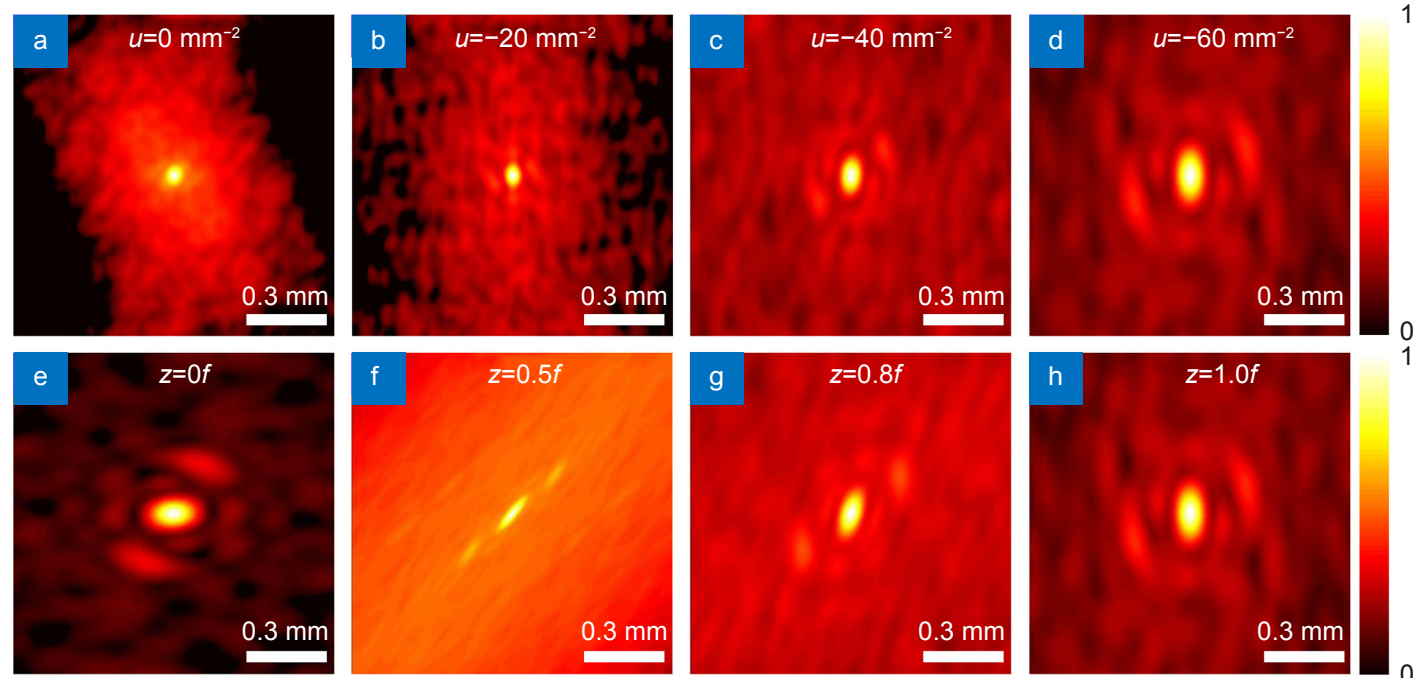

Fig. $4 \mid(\mathbf{a}-\mathbf{d})$ Experimental results of the modulus of the DOC $|\mu|$ in the focal plane with different strength factors $u$. (e-h) The modulus of the $\mathrm{DOC}|\mu|$ at different propagation distances $z$ after the lens with the CP strength factor $u=-60 \mathrm{~mm}^{-2}$. 

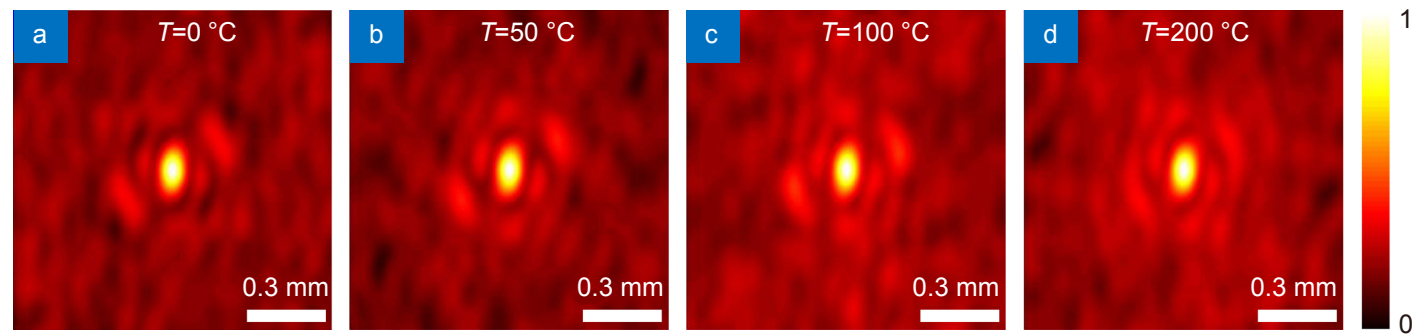

Fig. 5 | Experimental results of the modulus of the DOC $|\mu|$ in the focal plane at different temperatures of the HP. The strength factor of the $\mathrm{CP}$ is $u=-60 \mathrm{~mm}^{-2} . T=0{ }^{\circ} \mathrm{C}$ stands for the free space case.

Image transmission through free space and turbulent media via the modulus of the DOC

Inspired by the recovery of the modulus of the DOC of any Schell-model beam carrying a CP in the far field, we now employ this useful propagation feature for encoding a thin object's information in it. It is implied from Eq. (7) that the DOC is the Fourier transform of the intensity pattern $P(\boldsymbol{v})$ at the RGGD plane. If $P(\boldsymbol{v})$ is the desired image information, one only needs to obtain the DOC information in the receiver plane using a simple detection system shown in part II of Fig. 3 (distance $z$ is set as $f$ ). The image is then acquired through implementing the inverse Fourier transform of the measured DOC. Nevertheless, only the modulus of the DOC is obtained in the experiment through intensity correlation. To acquire the phase information, we adopt the Fienup's phase retrieval (FPR) algorithm ${ }^{45}$, which is based on an iterative algorithm to retrieve the phase from the known modulus of the DOC (see in the supplementary materials). Before implementing the FPR algorithm, the high background noise in the measured DOC is first filtered out and reduced.

Figure 6 illustrates the dependence of the strength factor of the CP on the quality of the recovered image in the focal plane of the lens (in the absence of turbulence). With the trivial $\mathrm{CP}$, the image information hidden in the DOC is lost when the beam propagates to the focal plane (in the far field) [see Fig. 6(a, b)]. The recovered image degenerates to a Gaussian-like spot. As the strength of the CP factor $|u|$ increases, the image gradually becomes clear. When the strength factor $u$ reaches $-60 \mathrm{~mm}^{-2}$, a high quality of the image can be achieved from the DOC measurement. The physical mechanism behind such a reconstruction was discussed in the previous section. It relies on the fact that the modulus of the DOC in the focal plane is exactly same as in the source plane.

In the presence of turbulence, the background noise in the measured DOC (see in Fig. 5) becomes significant with the increase of the HP temperature. In this case, clear images can also be reconstructed by means of FPR algorithms for the inverse Fourier transform of the DOC, see Fig. 7(d)-7(f). On comparison of the images obtained in the absence and in the presence of turbulence [see Fig. 6(d) and Fig. 7], only a slight deviation is found. To further demonstrate the robustness of the transmission of images in turbulence, a numerical analysis is also carried out with the help of the wave-optics simulation method and multi-phase screen method (see in the supplementary materials). The propagation scenario is the same as that in the part III in Fig. 3. The total propagation path from the source plane to the lens plane is $L=1$ $m$. Several random phase screens obeying Kolmogorov statistics are equally separated in the propagation path. The detector is placed in the focal plane to collect the instantaneous intensity from $\mathrm{L}_{6}$. In the numerical simulation, the power spectral density of turbulence is described by the von Kármán spectrum ${ }^{35}$
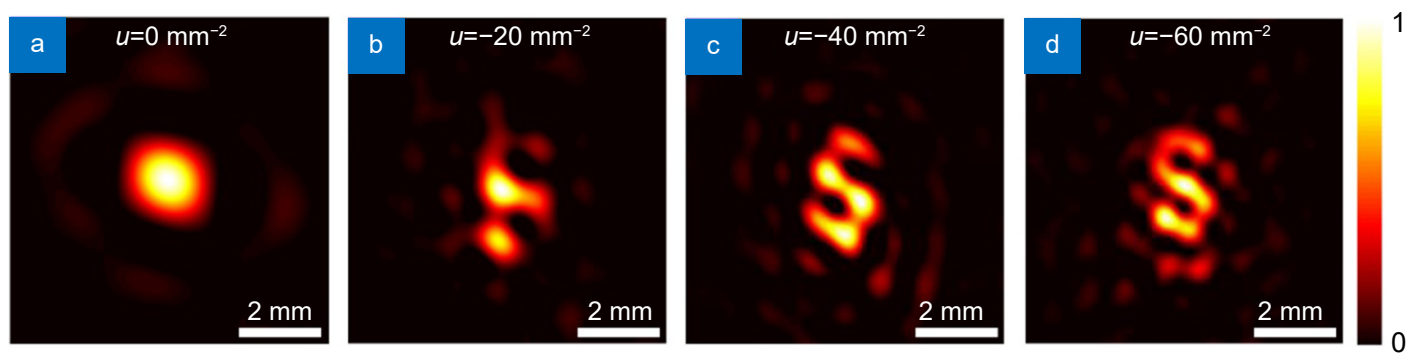

Fig. 6 | Experimental results of the dependence of the quality of the recovered image on the strength factor $u$ in the focal plane in free space. 

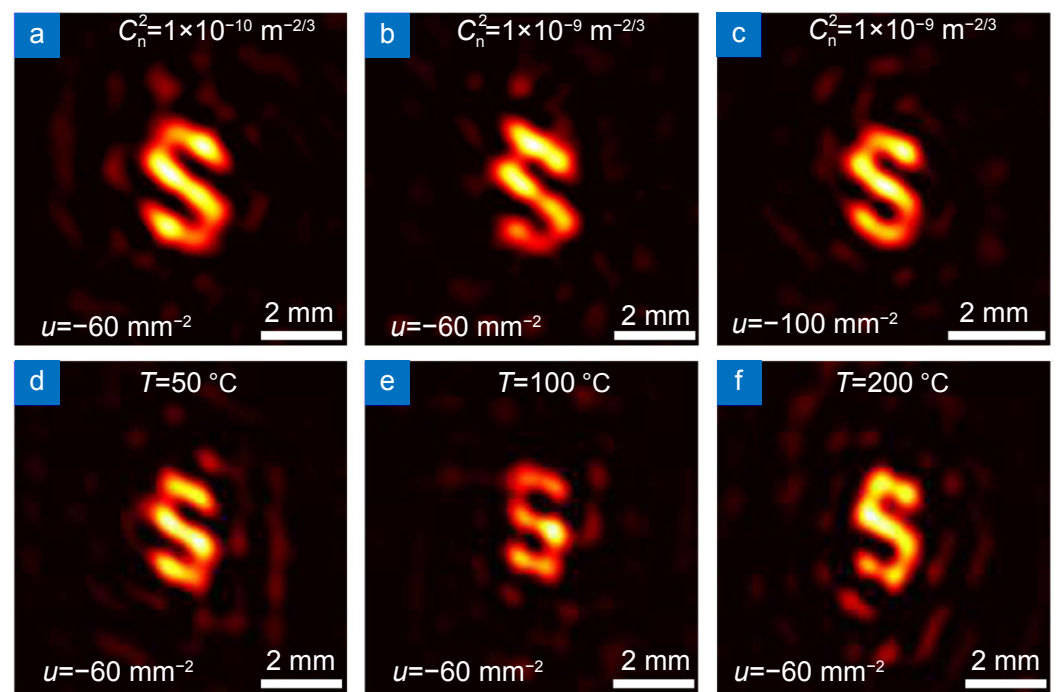

Fig. $7 \mid(\mathbf{a}-\mathbf{c})$ Simulation results of the reconstructed image in turbulence of different strength and with different CP strength factor $u$. (d-f) Experimental results of the reconstructed image at different temperatures with $u=-60 \mathrm{~mm}^{-2}$.

$$
\Phi_{n}(\kappa)=0.33 \mathrm{C}_{n}^{2}\left(\kappa^{2}+\kappa_{0}^{2}\right)^{-11 / 6} \exp \left(-\kappa^{2}+\kappa_{m}^{2}\right),
$$

where $\mathrm{C}_{n}^{2}$ is the structure constant, a measure of the strength of turbulence; $\kappa$ is the transverse spatial frequency; $\kappa_{\mathrm{m}}=5.92 l_{0}$ with $l_{0}$ being the inner scale, and $\kappa_{0}=$ $2 \pi / L_{0}$ with $L_{0}$ being the outer scale. The turbulence and beam parameters are chosen to be $l_{0}=1.0 \mathrm{~mm}, L_{0}=1.0 \mathrm{~m}$.

Figure $7(\mathrm{a})-7$ (c) presents the simulation results of the reconstructed image with different strengths of turbulence and CP strength factors. It can be seen that the high quality of image is acquired for $C_{n}^{2}=1 \times 10^{-10} \mathrm{~m}^{-2 / 3}$ and $u=-60 \mathrm{~mm}^{-2}$, while the quality decreases as the strength of turbulence increases [see in Fig. 7(b)]. One protocol to compensate the turbulence-induced degeneration is the further increase of the CP strength factor $|u|$. As shown in Fig. 7(c), the high quality image, as compared to that in Fig. 7(b), is obtained when the factor $u$ reaches $-100 \mathrm{~mm}^{-2}$ in a relatively strong turbulence. Figure $7(d)-7(f)$ exposes the experimental results of the retrieved image with the CP strength factor $u=-60 \mathrm{~mm}^{-2}$ at different temperatures. The images are well recovered for all of them, which well illustrates that the DOC has the robustness of the transmission of images in turbulence. At the same time, the image quality gets worse for the higher temperature, but it could be anticipated from the simulation examples that the image quality gets better with the increase of the CP strength factor $|u|$.

Another advantage of the image reconstruction via the DOC measurement is that one does not need to receive the whole beam cross-section in the receiver plane. In fact, a small part of the detection area suffices. As shown in Fig. 8(a), a typical instantaneous intensity pattern is captured by the CCD (GS3-U3-28S5M-C series, Point
Grey). The image in Fig. 8(b) is reconstructed from the measured DOC using the area indicated by the yellow square, and the image in Fig. 8(c) uses the area within the red square. It can be seen that the quality of two reconstructed images is almost the same. To ensure the quality of reconstructed image, the requirement for the size of the detection area should be 10 times larger than that of the DOC's pattern, otherwise the image information will be gradually lost. This feature substantially alleviates the need of the beam's perfect alignment in the receiver plane. After all, a small portion of the received beam contains the image information. The physical mechanism behind this phenomenon is that the light beam in the receiver plane retains the Schell-model characteristics either on propagation in free space or in the presence of turbulence. The modulus of the two-point DOC of the Schell-model field is only dependent on the separation between two points, and thus it is spatially shift-invariant, which means that any reference point in the detector area will acquire the same DOC information. As a result, one could recover the image with enough accuracy, provided that the DOC pattern is sufficiently narrow in the red area of the square.

\section{Conclusions}

In summary, we have studied the influence of the $\mathrm{CP}$ on the evolution of the DOC of Schell-model beams, having non-Gaussian profiles of their moduli, in a typical focusing optical system, being equivalent to free-space propagation to the far field. Our results reveal that, in the presence of the $\mathrm{CP}$, the modulus of the DOC during propagation gradually reverts to that in the source plane 

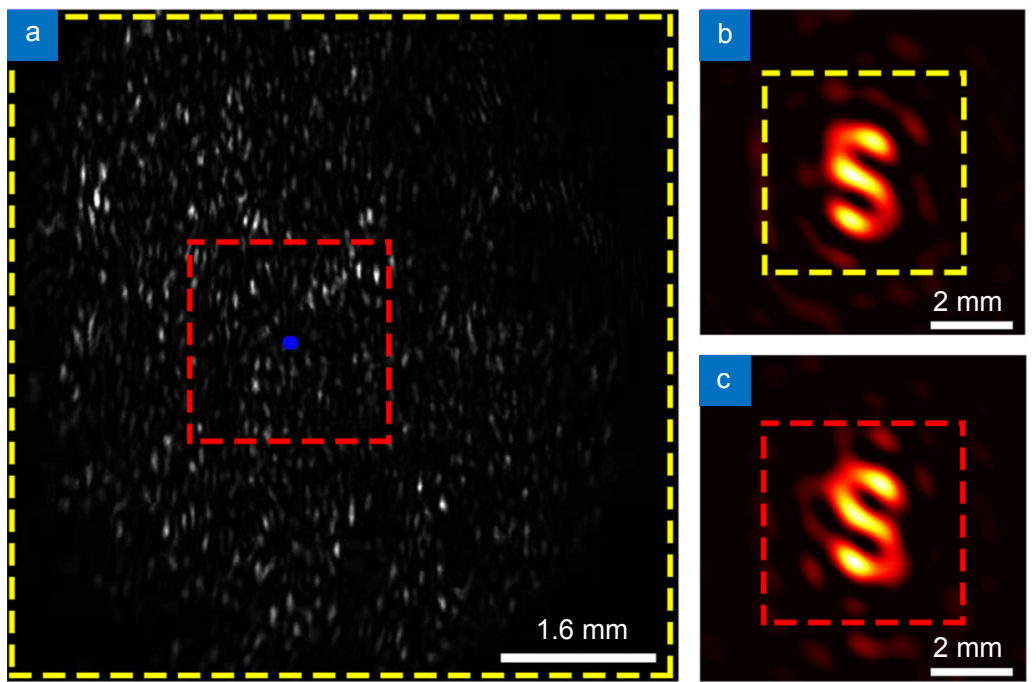

Fig. 8 | (a) A typical instantaneous intensity captured by the CCD. (b) Experimental results of the recovered image through the area surrounded by the yellow dashed square shown in subplot (a) covering $1440 \times 1440$ pixels. (c) The recovered image through the area surrounded by the red dashed square shown in subplot (a) covering $500 \times 500$ pixels.

after exhibiting structural changes. Moreover, it is shown that in the far field, the modulus of the DOC is exactly the same as that in the source plane if the strength of the $\mathrm{CP}$ factor satisfies a certain condition. This behavior is quite different from that of the Schell-model beams without the $\mathrm{CP}$, in which case the DOC irreversibly converts to other forms during propagation because of diffraction. Further, we experimentally demonstrate this peculiar propagation characteristic in free-space propagation and even in the presence of a moderate atmospheric turbulence.

Based on the discovered DOC's modulus recovery mechanism in the far field, an efficient far-field imaging system is proposed for the transmission of the image via spatial coherence engineering through atmospheric turbulence. With the help of Fienup's phase retrieval algorithms, a high quality image is reconstructed from the modulus of the far-field DOC. Through numerical simulation and experiment, we demonstrate that the proposed imaging scheme is quite resilient to the turbulence, and also greatly reduces the need for the beam alignment accuracy in the receiver plane, since only a small portion of the beam cross-section enables one to completely recover the image information. Our results shed new light on spatial coherence engineering for remote sensing and optical imaging in harsh environments.

\section{References}

1. Mandel L, Wolf E. Optical Coherence and Quantum Optics (Cambridge University Press, Cambridge, 1995).
2. Wolf E. Introduction to the Theory of Coherence and Polarization of Light (Cambridge University Press, Cambridge, 2007).

3. Cai YJ, Wang F, Zhao CL, Zhu SJ, Wu GF et al. Partially coherent vector beams: from theory to experiment. In Zhan QW. Vectorial Optical Fields: Fundamentals and Applications (World Scientific, Hackensack New Jersey, 2014).

4. Douglass KM, Sieben C, Archetti A, Lambert A, Manley S. Super-resolution imaging of multiple cells by optimized flat-field epi-illumination. Nat Photonics 10, 705-708 (2016).

5. Korotkova O, Gbur G. Applications of optical coherence theory. Prog Opt 65, 43-104 (2020).

6. Zanotto L, Piccoli R, Dong JL, Morandotti R, Razzari L. Singlepixel terahertz imaging: a review. Opto-Electron $A d v$ 3, 200012 (2020).

7. Gori $F$, Santarsiero M. Devising genuine spatial correlation functions. Opt Lett 32, 3531-3553 (2007).

8. Gori F, Ramírez-Sánchez V, Santarsiero M, Shirai T. On genuine cross-spectral density matrices. J Opt A: Pure Appl Opt 11, 085706 (2009).

9. Hyde IV MW, Bose-Pillai SR, Wood RA. Synthesis of non-uniformly correlated partially coherent sources using a deformable mirror. Appl Phys Lett 111, 101106 (2017).

10. Wang R, Zhu SJ, Chen YK, Huang HK, Li ZH et al. Experimental synthesis of partially coherent sources. Opt Lett 45, 1874-1877 (2020).

11. Zhu XL, Yu JY, Chen YH, Wang F, Korotkova O et al. Experimental synthesis of random light sources with circular coherence by digital micro-mirror device. Appl Phys Lett 117, 121102 (2020).

12. Lajunen $H$, Saastamoinen $T$. Propagation characteristics of partially coherent beams with spatially varying correlations. Opt Lett 36, 4104-4106 (2011).

13. Sahin S, Korotkova O. Light sources generating far fields with tunable flat profiles. Opt Lett 37, 2970-2972 (2012).

14. Korotkova O. Random Light Beams: Theory and Applications (CRC Press, Boca Raton, 2013).

15. Cai YJ, Chen YH, Wang F. Generation and propagation of partially coherent beams with nonconventional correlation functions: a review[Invited]. J Opt Soc Am A 31, 2083-2096 (2014).

16. Liang $\mathrm{CH}$, Wang $\mathrm{F}$, Liu XL, Cai YJ, Korotkova O. Experimental 
generation of cosine-Gaussian-correlated Schell-model beams with rectangular symmetry. Opt Lett 39, 769-772 (2014).

17. Liang $\mathrm{CH}$, Khosravi R, Liang XX, Kacerovská B, Monfared YE. Standard and elegant higher-order Laguerre-Gaussian correlated Schell-model beams. J Opt 21, 085607 (2019).

18. Ma LY, Ponomarenko SA. Free-space propagation of optical coherence lattices and periodicity reciprocity. Opt Express 23, 1848-1856 (2015).

19. Liu XL, Yu JY, Cai YJ, Ponomarenko SA. Propagation of optical coherence lattices in the turbulent atmosphere. Opt Lett 41, 4182-4185 (2016).

20. Liu X, Xia DN, Monfared YE, Liang CH, Wang F et al. Generation of novel partially coherent truncated Airy beams via Fourier phase processing. Opt Express 28, 9777-9785 (2020).

21. Zhou Y, Xu HF, Yuan YS, Peng J, Qu J et al. Trapping two types of particles using a Laguerre-Gaussian correlated Schellmodel beam. IEEE Photonics J 8, 6600710 (2016).

22. Liang $\mathrm{CH}$, Wu GF, Wang F, Li W, Cai YJ et al. Overcoming the classical Rayleigh diffraction limit by controlling two-point correlations of partially coherent light sources. Opt Express 25, 28352-28362 (2017).

23. Wang F, Chen YH, Liu XL, Cai YJ, Ponomarenko SA. Self-reconstruction of partially coherent light beams scattered by opaque obstacles. Opt Express 24, 23735-23746 (2016).

24. $\mathrm{Xu} \mathrm{ZH,} \mathrm{Liu} \mathrm{XL,} \mathrm{Chen} \mathrm{YH,} \mathrm{Wang} \mathrm{F,} \mathrm{Liu} \mathrm{L} \mathrm{et} \mathrm{al.} \mathrm{Self-healing}$ properties of Hermite-Gaussian correlated Schell-model beams. Opt Express 28, 2828-2837 (2020).

25. Lu XY, Shao YF, Zhao CL, Konijnenberg S, Zhu XL et al. Noniterative spatially partially coherent diffractive imaging using pinhole array mask. Adv Photonics 1, 016005 (2019).

26. Chen $\mathrm{YH}$, Ponomarenko SA, Cai YJ. Experimental generation of optical coherence lattices. Appl Phys Lett 109, 061107 (2016).

27. Huang ZF, Chen YH, Wang F, Ponomarenko SA, Cai YJ. Measuring complex degree of coherence of random light fields with generalized hanbury brown-twiss experiment. Phys Rev App/ 13, 044042 (2020).

28. Ma PJ, Kacerovská B, Khosravi R, Liang $\mathrm{CH}$, Zeng J et al. Numerical approach for studying the evolution of the degrees of coherence of partially coherent beams propagation through an ABCD optical system. App/ Sci 9, 2084 (2019).

29. Peng DM, Huang ZF, Liu YL, Chen YH, Wang F et al. Optical coherence encryption with structured random light. PhotoniX $\mathbf{2}$, 6 (2021).

30. Goodman JW. Statistical Optics (Wiley \& Sons, New York, 2000).

31. Miao JW, Charalambous P, Kirz J, Sayre D. Extending the methodology of X-ray crystallography to allow imaging of micrometre-sized non-crystalline specimens. Nature $\mathbf{4 0 0}$, 342-344 (1999).

32. Shechtman Y, Eldar YC, Cohen O, Chapman HN, Miao JW et al. Phase retrieval with application to optical imaging: a contemporary overview. IEEE Sig Process Mag 32, 87-109 (2015).

33. Soni NK, Vinu RV, Singh RK. Polarization modulation for imaging behind the scattering medium. Opt Lett 41, 906-909 (2016).

34. Zhang YB, Liu H, Cheng H, Tian JG, Chen SQ. Multidimen- sional manipulation of wave fields based on artificial microstructures. Opto-Electron Adv 3, 200002 (2020).

35. Andrews LC, Phillips RL. Laser Beam Propagation Through Random Media 2nd ed (SPIE Press, Bellingham, 2005).

36. Newman JA, Luo QE, Webb KJ. Imaging hidden objects with spatial speckle intensity correlations over object position. Phys Rev Lett 116, 073902 (2016).

37. Bertolotti J, van Putten EG, Blum C, Lagendijk A, Vos WL et al. Non-invasive imaging through opaque scattering layers. Nature 491, 232-234 (2012).

38. Katz O, Heidmann P, Fink M, Gigan S. Non-invasive singleshot imaging through scattering layers and around corners via speckle correlations. Nat Photonics 8, 784-790 (2014).

39. Wan LP, Zhao DM. Controllable rotating Gaussian Schell-model beams. Opt Lett 44, 735-738 (2019).

40. Liang G, Zhang HC, Fang L, Shou Q, Hu W et al. Influence of transverse cross-phases on propagations of optical beams in linear and nonlinear regimes. Laser Photonics Rev 14, 2000141 (2020)

41. Liang G, Wang Q. Controllable conversion between Hermite Gaussian and Laguerre Gaussian modes due to cross phase. Opt Express 27, 10684-10691 (2019).

42. Liu X, Li ZQ, Monfared YE, Liang $\mathrm{CH}$, Wang $\mathrm{F}$ et al. Flexible autofocusing properties of ring Pearcey beams by means of a cross phase. Opt Lett 46, 70-73 (2021).

43. Shen DH, Zhao DM. Measuring the topological charge of optical vortices with a twisting phase. Opt Lett 44, 2334-2337 (2019).

44. Ren Y, Wang C, Liu T, Wang ZK, Yin CZ et al. Polygonal shaping and multi-singularity manipulation of optical vortices via high-order cross-phase. Opt Express 28, 26257-26266 (2020).

45. Fienup JR. Reconstruction of an object from the modulus of its Fourier transform. Opt Lett 3, 27-29 (1978).

\section{Acknowledgements}

We are grateful for National Key Research and Development Program of China (2019YFA0705000); National Natural Science Foundation of China (11874046, 11947239, 11974218, 12004220, 91750201, 11904247, 12192254); Innovation Group of Jinan (2018GXRC010); Local Science and Technology Development Project of the Central Government (YDZX20203700001766); China Postdoctoral Science Foundation (2019M662424).

\section{Author contributions}

The design and theoretical analysis were carried out by Y. L. Liu, which was verified in experiment by Y. L. Liu and Y. H. Chen; The writing-original draft by Y. L. Liu and C. H. Liang; The writing-review and editing was carried out by O. Korotkova, C. H. Liang, F. Wang and Y. J. Cai; All authors revised the manuscript; The entire research was supervised by C. H. Liang, F. Wang and Y. J. Cai; All authors have read and agreed to the published version of the manuscript.

\section{Competing interests}

The authors declare no competing financial interests.

\section{Supplementary information}

Supplementary information for this paper is available at https://doi.org/10.29026/oea.2021.210027 